\title{
Assessing the Vulnerability of Replicated Network Services
}

\author{
George Dean Bissias Brian Neil Levine Ramesh K. Sitaraman \\ Dept. of Computer Science, Univ. of Massachusetts Amherst, USA \\ \{gbiss,brian,ramesh\}@cs.umass.edu
}

\begin{abstract}
Client-server networks are pervasive, fundamental, and include such key networks as the Internet, power grids, and road networks. In a client-server network, clients obtain a service by connecting to one of a redundant set of servers. These networks are vulnerable to node and link failures, causing some clients to become disconnected from the servers. We develop algorithms that quantify and bound the inherent vulnerability of a clientserver network using semidefinite programming (SDP) and branch-and-cut techniques. Further, we develop a divide-and-conquer algorithm that solves the problem for large graphs. We use these techniques to show that: for the Philippine Power Grid removing just over 6\% of the transmission lines will disconnect at least 20\% but not more than $50 \%$ of the substations from all generators; on a large wireless mesh network disrupting $5 \%$ of wireless links between relays removes Internet access for half the relays; even after any $16 \%$ of Tier 2 ASes are removed, more than $50 \%$ of the remaining Tier 2 ASes will be connected to the Tier 1 backbone; when 300 roadblocks are erected in Michigan, it's possible to disconnect 28-43\% of the population from all airports.
\end{abstract}

\section{INTRODUCTION}

A client-server network consists of clients and servers where each client obtains a critical service by connecting over the network to a server chosen from a redundant set. The key aspects of several real-world networks can be modeled as client-server networks, with suitable definitions for clients and servers. For instance, a wireless

This work was supported in part by NSF Awards CNS0519894 and CNS-0519881.

Permission to make digital or hard copies of all or part of this work for personal or classroom use is granted without fee provided that copies are not made or distributed for profit or commercial advantage and that copies bear this notice and the full citation on the first page. To copy otherwise, to republish, to post on servers or to redistribute to lists, requires prior specific permission and/or a fee.

ACM CoNEXT 2010, November 30-December 3 2010, Philadelphia, USA. Copyright 2010 ACM 1-4503-0448-1/10/11 ...\$10.00. mesh consists of mobile users (clients) accessing the wired Internet by connecting to one of the gateways (servers). In the Internet context, Tier-2 autonomous systems (clients) connect to the Internet backbone by peering with one of the Tier-1 autonomous systems (servers). In a power grid, each substation (client) connects to one of the power generators (servers) to obtain electricity. And, people (clients) travel to airports and other critical service infrastructure (servers) using the road network.

Client-server networks are vulnerable to node and link failures, and clients that are unable to connect to no server at all are denied service. Failures may be caused by both genuine malfunction and intentionally targeted attacks. Even though servers are typically deployed in a distributed and redundant fashion to enhance service availability, a targeted set of failures can cause serious denial of service to clients. Network attacks and failures are common in a wide variety of networks. Wireless networks are susceptible to numerous attacks including DOS, spoofing attacks, and physical tampering $[21,24,32,33]$. In wired networks, fiber optic cables and other media are conspicuously susceptible to intentional or inadvertent destruction $[8,16,25]$. Internet Autonomous Systems (ASes) are vulnerable to BGP protocol exploits that have been documented by numerous researchers for years $[20,23]$. Power grids are crucial to modern infrastructure as illustrated by the major blackout in North America in 2003 and many point out targeted (terrorist) attacks are a significant concern $[6,7,30]$. The industry consensus is a need to identify attack and failure scenarios before they happen [6, 26]. Finally, there is significant concern for the national highway system. A recent study identified eight unrelated suspicious events that seemed to indicate terrorist attention to major pieces of domestic highway infrastructure [19] via roadside or vehicle-borne improvised explosive devices (IEDs). To address these concerns, the U.S. Government Accountability Office calls for individual vulnerability assessments on the nationally critical Tier-2 structures list [9].

All of the above examples of real-world client-server 
networks underscore the importance of studying the effect of failures and attacks on service availability for clients. Our specific goal is to assess the intrinsic vulnerability of a client-server network by computing a quantitative relationship between the number of compromised or failed network resources and the number of clients who are denied service.

\subsection{Problem Statement}

A client-server network can be modeled as a graph $G=(S \cup C, E)$, where $S$ is a set of server vertices, $C$ is a set of client vertices, and $E$ is the set of edges. Each server is equally capable of providing the required service and each client accesses the service by connecting to any one of the servers in $S$. Taking the power grid as an example, the set $S$ consists of generator nodes that serve electricity and the set $C$ consists of substation nodes that receive and distribute it to customers. If any substation is disconnected from all generators, then it and all of its customers will lose power. Wireless mesh networks can also be modeled as client-server graphs where set $S$ is the small number of gateways that are directly connected to the Internet, while the set $C$ consists of the large number of relays that connect to gateways or other relays. Internet connectivity is established for any given relay only when there exists some path to a gateway.

Our problem can be formally defined as follows. We define a service path for a client vertex $v$ as any path in $G$ that begins at $v$ and ends at some server vertex. Client $v$ is said to be serviceable if and only if it has a service path. Let $\operatorname{Conn}(G)$ be the number of serviceable clients in $G$. Let $G \ominus R$ be the graph $G$ with elements $R \subseteq S \cup C \cup E$ removed; i.e., $G \ominus R$ is the graph that results when the vertices and edges in $R$ fail $^{1}$. We focus on solving the following problem in the context of real networks.

\section{PROBLEM: $\operatorname{MinConn}(G, n, m)$}

- Given: client-server graph $G=(S \cup C, E)$ and $n, m \in \mathbb{R}$.

- Find: minimum value of $\operatorname{Conn}(G \ominus(A \cup B))$, over all $A, B$, where $A \subseteq C,|A|=n$, and $B \subseteq E,|B|=m$.

Note that $\operatorname{Min} \operatorname{Conn}(G, n, m)$ is simply the number of serviceable clients in $G$ in the worst scenario of $n$ client vertex failures and $m$ edge failures.

We also solve a weighted version of the above problem that we call WeightedMinConn $(G, n, m)$ where each $v \in$ $C$ is assigned a weight $w[v]$. The objective is to minimize the weighted sum of the serviceable clients in $G \ominus(A \cup$ $B$ ), where $A \subseteq C$ and $B \subseteq E$, with $\sum_{v \in A} w[v] \leq n$ and $|B|=m$. The weighted variant of the problem is

\footnotetext{
${ }^{1}$ We assume that failed vertices and links cannot be used for any purpose, and hence may be removed.
}

useful in our road network analysis where each vertex represents a geographical area and is weighted by its population density.

\subsection{Our Results}

Our main contributions are as follows. We formalize the notion of vulnerability in client-server networks by defining the MinConn problem and its weighted variant. Since solving the MinConn problem exactly is NP-Hard (see Bissias et al. [10]), we develop algorithms that derive upper and lower bounds. We provide the first efficient algorithms for bounding MinConn for both edge and client vertex removal (separate or combined) using semidefinite programming and a branch-and-cut technique. Further, we extend these techniques to large graphs using a divide-and-conquer technique. Finally, we demonstrate the wide applicability of our techniques on real-world data sets, providing quantitative results.

Our real-world analysis includes (i) a residential wireless mesh network, (ii) the power grid of the Philippines, (iii) the Internet AS level graph, and (iv) the highway systems in Iowa and Michigan. We chose these datasets for their importance, diversity, and size. In particular, the AS and highway system graphs are large at over 5,000 and 1,500 vertices, respectively.

For the mesh network, roughly half the relays are disconnected from all gateways after removing less than $5 \%$ of the links, while removing less than $18 \%$ of the relays can disconnect more than $70 \%$ of the remaining relays. For the power grid, removing just over $6 \%$ of the transmission lines will disconnect at least $20 \%$ but not more than $50 \%$ of the substations from all generators, while almost $50 \%$ of the substations can be disconnected from all generators after removing a certain $5 \%$ of the other substations. In the AS graph, even when greater than $16 \%$ of the Tier- 2 nodes are removed, more than half the Internet remains connected. For airport connectivity, after 200 roadblocks are erected it is possible to disconnect $15 \%$ to $50 \%$ of the population from every airport in Iowa. In Michigan, only $28 \%$ to $43 \%$ of the population will maintain airport connectivity after erecting 300 road blocks.

\subsection{Related Work}

A traditional approach to studying the vulnerability of a complex network is to use graph partitioning techniques such as finding graph separators of small width [28]. An $\alpha$-separator of a graph $G=(V, E)$ is a collection of either edges or vertices whose removal separates a graph into two disconnected subgraphs, each having size at most $\lceil(1-\alpha)|V|\rceil$. The number of edges or vertices in the cut is called the separation width. Finding either an $\alpha$-edge-separator or $\alpha$-vertex-separator of minimum width is NP-hard. Bui and Jones have shown it is NP-hard even to find a good approxima- 
tion to the $\alpha$-separator problem [11]. The objective of graph separation is to completely partition the graph into appropriately-sized pieces by removing vertices and edges. In contrast, our goal is not to partition the graph completely, but to study the manner in which service availability degrades as a function of the number of failed components.

Even though standard graph partitioning does not appear to be directly applicable, some of the techniques developed in that context have been key to our approach. Specifically, Wolkowicz and Zhao [31] developed techniques for using semidefinite programming for graph partitioning. We utilize some of those ideas in our algorithm, specifically the notion of a lift matrix to represent our block decomposition of the client-server graph.

Numerous works experimentally evaluate the vulnerability of specific networks or graph families. However, they do not consider a client-server formulation with a focus on service availability as we do here. Neither do they develop algorithms that analyze worst-case failure scenarios as in our work. Ding et al. [14] used spectral techniques to decompose the web graph. Magoni and Zhou et al. $[22,34]$ independently analyzed the Internet AS graph and showed that by removing approximately $3 \%$ of the ASes (including Tier-1's and Tier-2's) the size of the largest connected component decreases by a factor of 3. Guillaume et al. [17] evaluated the vulnerability of scale free and Erdos-Renyi (ER) graphs subjected to a randomized link attack. In this context, they found that scale free graphs have approximately the same robustness as the ER graphs. Flaxman et al. [15] provided a framework for strengthening existing graphs by supplementing them with regular subgraphs. Cohen et al. [12] provided a modification to classical percolation theory in order to study the effects of removing vertices with highest degree, and demonstrated that even though scale-free graphs are robust to random failure they are highly sensitive to targeted attack.

\subsection{Roadmap}

In Section 2, we provide an algorithm that uses semidefinite programming (SDP) and branch-and-cut techniques to bound the values of $\operatorname{Min} \operatorname{Conn}(G, n, m)$ for arbitrary client-server networks. Further, we apply these techniques to assess the vulnerability of two real-world networks: a wireless mesh network and the power grid of the Philippines. In Section 3, we extend the SDP-based approach to larger graphs by using a divide-and-conquer heuristic. Further, we apply this approach to two larger real-world networks: the Internet AS graph, and the road networks of Iowa and Michigan. Finally, in Section 4 , we offer concluding remarks.

\section{AN SDP-BASED ALGORITHM}

The goal of this section is to derive upper and lower

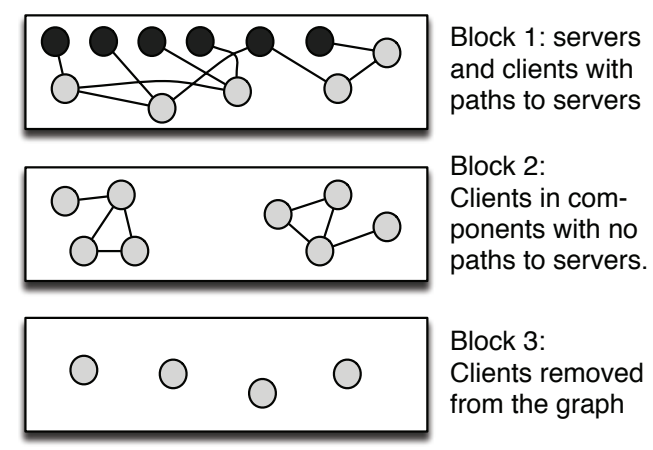

Figure 1: Separating a Client-Server Graph into blocks

bounds on $\operatorname{Min} \operatorname{Conn}(G, n, m)$ by means of a semidefinite program (SDP). For clarity, the SDP is translated from a conceptually simple, but numerically unstable, Quadratically Constrained Quadratic Program (QCQP) formulation. Fundamental to our approach is a block representation for $G$ that separates serviceable, nonserviceable, and entirely removed clients.

\subsection{QCQP Formulation}

Let $A$ be the adjacency matrix of $G=(S \cup C, E)$, and for convenience label $V \equiv S \cup C$. Further, for any $V^{\prime} \subseteq V$, let $G\left(V^{\prime}\right)$ denote the induced subgraph of $G$ whose vertex set is $V^{\prime}$ and whose edge set is the subset of $E$ consisting of those edges with both ends in $V^{\prime}$. A block $B$ is any (possibly empty) subset of $V$ such that $G(B)$ is disconnected from $G(V \backslash B)$, but neither subgraph $G(B)$ nor $G(V \backslash B)$ are necessarily connected individually. We designate three blocks $B_{1}, B_{2}$, and $B_{3}$, with $\left|B_{i}\right|=b_{i}$. The block indicator matrix for $G$ is a $|V| \times 3$ matrix $X$, where

$$
X(i, j)=\left\{\begin{array}{ll}
1, & \text { node } i \in B_{j} \\
0, & \text { otherwise }
\end{array} .\right.
$$

It follows that $\sum_{i} X(i,:)=1$ and $\sum_{j} X(:, j)=b_{j}$, where ":" denotes any cell along that dimension (as in Matlab).

We next seek to frame the MinConn problem in terms of the three blocks we have created above, illustrated in Fig. 1. These blocks will correspond to a segmentation of serviceable, non-serviceable, and removed clients in $G$. Specifically, the first block is the server block, to which all server vertices are fixed, and clients with paths to these servers are placed. Clients without paths to servers are placed in the second block. Finally, the third block is the null block, which is where clients that have been removed from the graph are placed. The set of removed vertices and edges are called the vertex and edge cuts, and they are labeled $\mathcal{C}_{V}$ and $\mathcal{C}_{E}$ respectively. We have the following QCQP formulation.

- Given: client-server graph $G=(S \cup C, E)$ and $n, m \in \mathbb{R}$.

- Find: minimum $\|X(:, 1)\|$ such that, 
(1) $X(i, 1)=1, \forall i \in S$

(All servers in Block 1)

(4) $\left|\mathcal{C}_{E}\right| \leq m$.

(5) $X(i, j) \in\{0,1\}$

(Vertex Count)

(Vertex Cut Size)

(Edge Cut Size)

$(0-1$ property)

Note that $\mathcal{C}_{V} \equiv B_{3}$ so

$$
\left|\mathcal{C}_{V}\right|=\sum_{i=1}^{|V|} X(:, 3) .
$$

Finding an expression for $\left|\mathcal{C}_{E}\right|$ is just a bit more complicated. We begin with a short digression. Vector $X(:, j)$ indicates the vertex membership of block $j$. That is to say, $X(i, j)=1$ if vertex $i$ is in block $j$ and is 0 otherwise. For an arbitrary matrix $M$, the quantity $X(:, j)^{T} M X(:, j)$ gives the sum of entries in the submatrix indicated by $X(:, j)$. Define $D$ to be the matrix with row sums of adjacency matrix $A$ along its diagonal, and further define the Laplacian matrix $L=D-A$. The quantity $X(:, j)^{T} D X(:, j)$ gives twice the total number of edges incident to vertices indicated by $X(:, j)$, and $X(:, j)^{T} A X(:, j)$ gives twice the number of edges fully contained in block $j$. It follows that $X(:, j)^{T} L X(:, j)$ gives twice the number of edges incident to, but not fully contained in, block $j$.

To quantify $\left|\mathcal{C}_{E}\right|$, we seek to bound the number of edges between blocks 1 and 2, that is, all edges connecting the two blocks. This quantity is captured by

$$
\left|\mathcal{C}_{E}\right|=\frac{1}{2} \sum_{j=1}^{2} X(:, j)^{T} L X(:, j)-\frac{1}{2} X(:, 3)^{T} L X(:, 3) .
$$

The first term gives the total number of edges that lie between any of the three blocks. The second term subtracts the number of edges that pass between the null block and the others.

\subsection{SDP Formulation}

The QCQP formulation cannot be solved directly because there is no mechanism to enforce orthogonality between block vectors; hence there is no way to force each vertex into a single partition. Directly enforcing orthogonality is not possible in a QCQP. Therefore, we must graduate to a richer language, that of semidefinite programming. Every semidefinite program with an $N \times$ $N$ constraint matrix can be solved in time $O\left(N^{3}\right)$ [18]. A semidefinite program is any problem of the form

Minimize $C \bullet Z$ subject to $A \bullet Z=b$ and $Z \succeq 0$,

where $A \bullet B=\operatorname{trace}\left(A^{T} B\right)=\sum_{i j} A_{i j} B_{i j}$ is the Frobenius inner product and $Z \succeq 0$ indicates that $Z$ is positive semidefinite.

The Frobenius inner product gives the componentwise product of two matrices. So, for example, the $i j^{\text {th }}$ entry of $A \bullet Z$ is equal to the product of the $i j^{\text {th }}$ entry of $A$ and the $i j^{\text {th }}$ entry of Z. Our goal is to implement each of the constraints in the QCQP formulation in terms of a semidefinite optimization variable. To that end, let $\operatorname{vec}(X)$ be the vector formed by concatenating the columns of $X$. Furthermore, let

$$
y=\left[\begin{array}{c}
1 \\
\operatorname{vec}(X)
\end{array}\right] \text {. }
$$

Define the lift matrix associated with $X$ as $Y=y y^{T}$. Wolcowicz and Zhao [31] were the first to use this representation in the context of graph decomposition. First order terms are found along the first row and column, while second order terms are easily extracted from the remainder of the matrix. For notational simplicity, we number the rows and columns of $Y$ beginning with index zero. We will also use the function $\phi(i, j)=i+|V|(j-1)$, which maps the entry $X(i, j)$ to its corresponding entry in $\operatorname{vec}(X(i, j))$. The matrix $Y$ comprises all pairwise products of block indices of the form $X\left(i_{1}, j_{1}\right) X\left(i_{2}, j_{2}\right)$, as well as single indices $X(i, j)$. Term $X\left(i_{1}, j_{1}\right) X\left(i_{2}, j_{2}\right)$ indicates that vertex $i_{1}$ appears in block $j_{1}$ and vertex $i_{2}$ appears in block $j_{2}$. We have,

$$
Y\left(\phi\left(i_{1}, j_{1}\right), \phi\left(i_{2}, j_{2}\right)\right)=X\left(i_{1}, j_{1}\right) X\left(i_{2}, j_{2}\right),
$$

while

$$
Y\left(0, \phi\left(i_{1}, j_{1}\right)\right)=Y\left(\phi\left(i_{1}, j_{1}\right), 0\right)=X\left(i_{1}, j_{1}\right) .
$$

Now suppose that $B\left(\phi\left(i_{1}, j_{1}\right), \phi\left(i_{2}, j_{2}\right)\right)=\beta$ for some matrix $B$, then the term $\beta X\left(i_{1}, j_{1}\right) X\left(i_{2}, j_{2}\right)$ will appear in row $\phi\left(i_{1}, j_{1}\right)$ and column $\phi\left(i_{2}, j_{2}\right)$ of matrix $B \bullet Y$. Constraints can thus be built as scaled single and pairwise products of block indices.

THEOREM: There exists an SDP whose solution is at most the solution of our QCQP formulation.

PROOF: It will suffice to rewrite the objective and each of the constraints of our QCQP formulation as an SDP in terms of the lift matrix $Y$, where $Y$ is relaxed to the space of all semidefinite matrices. To that end let $\nu \equiv|V|$, and define $1_{i \times j}$ and $0_{i \times j}$ to be the matrices of all ones and zeros, respectively, and having size $i \times j$. The operator diag is defined as in Matlab so that it either extracts the diagonal of a matrix or forms a diagonal matrix from a vector. Also, let $I_{i \times i}$ be the identity matrix of size $i \times i$ and the symbol " $\otimes$ " denote the Kronecker Product of two matrices. The following is an SDP formulation.

PROGRAM: $\operatorname{Min} \operatorname{Conn} S D P(G, n, m)$

- Given: graph $G=(S \cup C, E)$ and $n, m \in \mathbb{R}$.

- Find: minimum $\mathcal{O} \bullet Y$ such that, 
(1) $\mathcal{F}_{i 1} \bullet Y=1, \forall i \in S$.

(Servers in Block 1)

(2) $\mathcal{S} \bullet Y=\nu$.

(Vertex Count)

(3) $\mathcal{V} \bullet Y=n$.

(Vertex Cut Size)

(4) $\mathcal{E} \bullet Y=m$.

(Edge Cut Size)

(5) $\mathcal{A}_{i j} \bullet Y=1, \forall i, j$.

(0-1 property)

(6) $\mathcal{U} \bullet Y=1$.

(Upper Left Equal 1)

(7) $\mathcal{Z}_{i} \bullet Y=1, \forall i$.

(Sum Vertex Values is 1)

where

$$
\begin{aligned}
& -\mathcal{O}=\operatorname{diag}\left(\left[\begin{array}{ccc}
0 & 1_{1 \times \nu} & 0_{1 \times 2 \nu}
\end{array}\right]\right) \\
& -\mathcal{S}=\operatorname{diag}\left(1_{|y| \times|y|}\right) \text { except that } \mathcal{S}(0,0)=0 \\
& -\mathcal{V}=\operatorname{diag}\left(\left[\begin{array}{ccc}
0 & 0_{1 \times 2 \nu} & 1_{1 \times \nu}
\end{array}\right]\right) \\
& \text { - } \mathcal{E}=\left[\begin{array}{ccc}
0 & 0_{1 \times 2 \nu} & 0_{1 \times \nu} \\
0_{2 \nu \times 1} & \frac{1}{2} I_{2 \times 2} \otimes L & 0_{2 \nu \times \nu} \\
0_{\nu \times \nu} & 0_{\nu \times 2 \nu} & -\frac{1}{2} L
\end{array}\right] \\
& \text { - } \mathcal{A}_{i j}=0_{|y| \times|y|} \text { except that } A_{i j}(\phi(i, j), 0)=1 \text { and } \\
& A_{i j}(\phi(i, j), \phi(i, j))=-1 \\
& \text { - } \mathcal{F}_{i j}=0_{|y| \times|y|} \text { except that } F_{i j}(\phi(i, j), \phi(i, j))=1 \\
& \text { - } \mathcal{U}=\operatorname{diag}\left(1_{|y| \times|y|}\right) \text { except that } \mathcal{U}(0,0)=1 \\
& \text { - } \mathcal{Z}_{i}=0_{|y| \times|y|} \text { except that } Z_{i}(\phi(i, j), \phi(i, j))=1 \text { for } \\
& \text { all } j \in\{1,2,3\}
\end{aligned}
$$

Constraints for the edge cut and the 0-1 property were first introduced by Wolcowicz and Zhao [31], but we have modified the edge cut constraint to subtract the number of edges passing to the null block. The last two constraints, in bold, have been introduced for the purposes of numerical stability: if $Y$ is drawn from the outer product space of $y$ (from equation 4 ), then it should always be the case that $Y(0,0)=1$, and if $\mathrm{Y}$ represents a valid partition, then the sum of diagonal entries corresponding to the placement of vertex $i$ in each block should add to 1 . Note that when translating constraints for mathematical programs, it is often necessary to use decompositions that involve simpler and sometimes redundant (in the space of binary vectors) constraints.

\subsection{Solving the SDP Formulation}

Semidefinite Programs can be solved by standard software suites. We use SDPT3 for MATLAB. This yields a tight lower bound for very small graphs. However, as the size of the input graph increases, the solver fails to assign each vertex to a single block. Instead, vertices are fractionally assigned to all blocks. We address this issue by fixing a small number of vertices to a single block as part of a branch-and-cut algorithm. Our entire solution procedure is as follows.

PROCEDURE: $\operatorname{Min} \operatorname{Conn} B C(G, n, m)$

- Solve Program MinConnSDP $(G, n, m)$ using SDPT3.

- Use branch-and-cut procedure to find a lower bound.
- Derive Upper bound by rounding.

The next section provides the details of this approach.

\subsubsection{Lower Bound via Branch and Cut}

Let $\mathcal{S}$ be the value of the semidefinite programming solution from Section 2.2. $\mathcal{S}$ offers a lower bound on the optimal solution to $\operatorname{Min} \operatorname{Conn}(G, n, m)$. This bound can be strengthened by fixing a small set of vertices $T$, each to one of the three blocks. In doing so, we move the semidefinite variable $Y$ closer to being a binary matrix, which shrinks the search space and ultimately leads to a tighter bound. On the other hand, we cannot be certain that the resulting solution is optimal because it's possible that the optimal solution does not admit the placement we have chosen. However, if we evaluate all $|Y|^{3}$ possible permutations of vertices $T$, then we can be certain that the lowest solution among them is no greater than optimal.

We choose the vertices of $T$ one-by-one. The first vertex, $v_{0}$, is chosen to be that whose placement is most ambivalent in $\mathcal{S}$ - that is, closet to one-third in each block. For each branch on $v_{0}$, the next vertex, $v_{1}$, is chosen as the most ambivalent vertex assignment in the given branch. This process is continued until the entire set $T$ is formed. Any given vertex $i$ is fixed to block $j$ by adding the constraint $\mathcal{F}_{i j} \bullet Y=1$ to the SDP. To stem the exponential growth of solution branches, we can eliminate any branch that places more than $m$ pairs of connected vertices in different blocks (not including the null block).

\subsubsection{Upper Bound via Rounding}

Our goal is to round some solution matrix $Y^{*}$ from the solution tree formed via branch-and-cut into a concrete solution to Problem MinConn $(G, n, m)$. In principle, any partial solution from the tree is a valid candidate, but in practice we find it difficult to round most solutions while simultaneously keeping the objective value low and satisfying the edge cut constraint. So we try multiple solution matrices and choose the best rounded solution among them. The best candidates are those matrices $Y^{*}$ that are nearly binary, and for small graphs these are readily found in the solution tree. However, for large graphs, a binary solution does not emerge immediately, so we choose the best branches at the last level of the solution tree and follow them exclusively until a nearly binary matrix $Y^{*}$ is found.

We now proceed under the assumption that a suitable matrix $Y^{*}$ has been identified. Given $Y^{*}$, define $Z^{*}$ to be the block matrix formed by extracting the diagonal of $Y^{*}$ and being fashioned in a manner analogous to the block matrix $X$. Matrix $Z^{*}$ is therefore an $|V| \times 3$ matrix with each row corresponding to a single vertex and possessing net weight 1 . Entry $Z^{*}(i, b)$ indicates the presence of vertex $i$ in block $b$, which may be fractional. 
Rounding $Z^{*}$ means rounding each row so that a single column in that row is equal to 1 . This constitutes an unambiguous assignment for each vertex.

Finally, we apply the Kernighan-Lin Algorithm [28] to reduce the number of edges in the cut. The the Kernighan-Lin Algorithm is an iterative procedure that begins by swapping every possible pair of vertices $\left(v_{0}, v_{1}\right)$ one-at-a-time where $v_{1}$ is in block 1 and $v_{2}$ is in block 2 . The pair that lowers the edge cut by the most is chosen and the vertices in the pair are fixed to their respective blocks. This procedure is repeated until there are no unfixed vertices remaining in one or both blocks. This procedure is attractive because it preserves the objective value and is known to produce small edge cuts when the initial partition is a good one. If we branch deeply enough with Procedure MinConnBC, then we will have a good initial partition. Unfortunately, it's difficult to discover a priori the necessary branch depth.

\subsection{Empirical Evaluation}

We compared the lower and upper bounds found by executing Procedure MinConnBC $(G, n, m)$ in two different real world networks: a wireless mesh network from the Net Equality Project and the Philippine Power Grid.

\subsubsection{Net Equality Wireless Mesh Network}

In the context of a wireless mesh network, vertex removal implies that a relay has been disabled while link removal implies that the connection between two nodes has been disrupted. Here, a relay node is a client and gateway node a server; our trace had no information about the number of users (laptops, desktops, etc.) connected to clients. We investigated how the number of serviceable clients decreases as other clients and links are removed from the network.

The Net Equality Project [3] works to provide Internet access to low-income communities where residents live in dwellings within close proximity, such as apartment complexes. They deploy a small number of gateway nodes that provide direct access to the Internet and relatively inexpensive Meraki Mini access points (relays) around the community, where most relays are multiple hops from gateways. A resident has access to the Internet wherever he/she is in range of either a gateway or a relay that is ultimately connected to a gateway. We analyzed a snapshot of the Hacienda CDC housing development taken in 2007. At the time, 69 relays and 2 gateways were connected by 279 links serving approximately 1,200 residents. We studied the removal of up to 18 clients and 20 links in the Net Equality Network.

Figure 2 shows the degradation of client serviceability in the network as links are removed. The blue triangles give a lower bound on the number of relays connected to the Internet after the indicated number links have been removed. Circles in red provide a complimentary

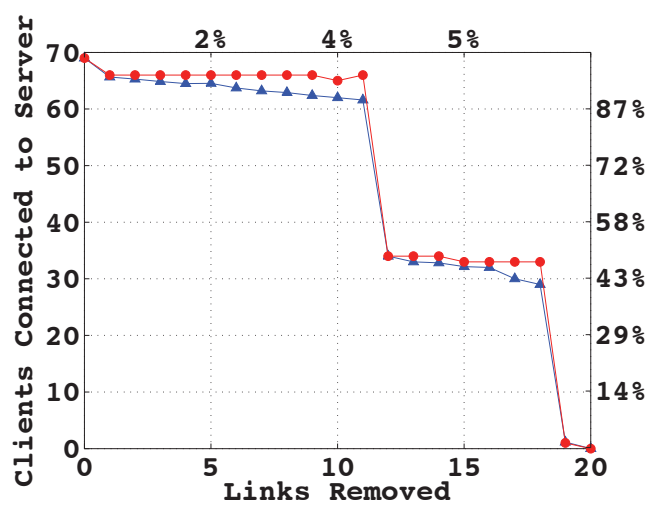

Figure 2: Wireless mesh connectivity (link removal)

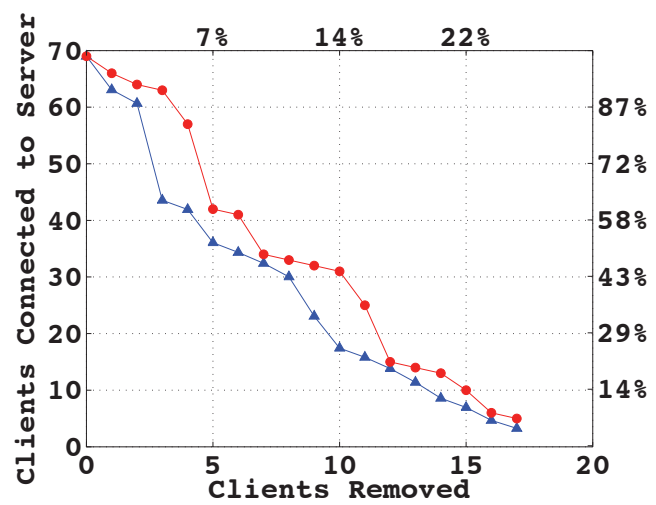

Figure 3: Wireless mesh connectivity (relay removal)

upper bound on the number of serviceable clients. These values correspond to partial (lower bound) and complete (upper bound) block assignments for each vertex. Two major features dominate Figure 2, the first is a severe drop in client serviceability after allowing 12 links to be removed. The second is another drop when 19 links can be removed. These points coincide with events where large subgraphs are finally disconnected from both gateways. This behavior provides valuable insight into the fault tolerance and attack vulnerability of the Net Equality graph. Communication difficulties between a handful of relays will not usually cause a major disruption, but degradation is not smooth, in general.

Figure 3 offers a view of client removal. Again, triangles in blue indicate a lower bound on the number of remaining clients that are serviceable when the given number of clients have been removed. Circles in red offer a complimentary upper bound. In contrast to the link removal problem, client removal exhibits a much more uniform decline in serviceability. However, while the rate of deterioration is roughly uniform, it is much more severe for the same number clients removed as links. For example, removing 8 clients can disconnect as many as 39 clients from all servers, while removing 8 links will disconnect no more than 10 clients. 


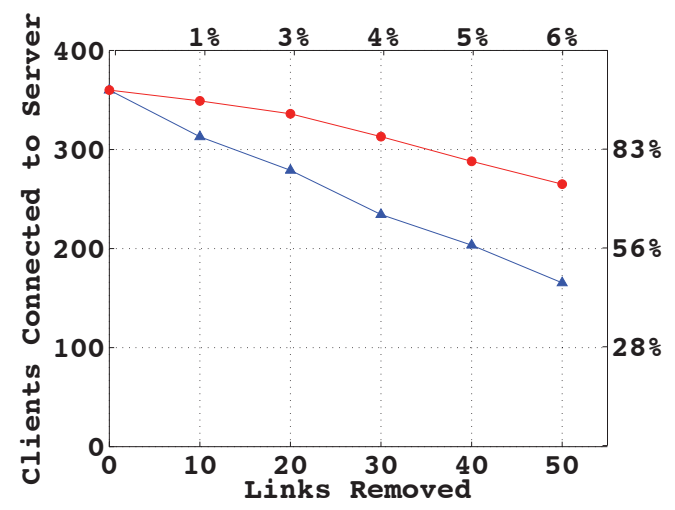

Figure 4: Power Grid connectivity (link removal)

\subsubsection{Philippine Power Grid}

We also analyzed a network representing the Power Grid of the Philippines from 2006 [4]. The Grid comprised 44 power generators and 360 substations connected by 796 transmission lines. We modeled the Grid as a client-server graph with power generators corresponding to servers, substations corresponding to clients, and transmission lines corresponding to edges. We studied, independently, the effect of removing up to 50 transmission lines (links) or 50 substations (clients).

Figure 4 shows how power service degrades as the number of links removed increases. The lower bound departs somewhat from the upper, but does serve to establish that removing as many as 50 of transmission lines will not disconnect more than $50 \%$ of the substations. On the other hand, the plot also indicates that it is possible to disconnect roughly $20 \%$ of the substations after removing some 50 transmission lines.

Figure 5 shows client serviceability degradation with client removal. The most noticeable feature is the greater impact client removal has on client serviceability compared with link removal. The bounds also depart from each other more gradually than in the case of link removal. Almost $50 \%$ of the substations can be disconnected by strategically removing just 20 other substations ; i.e., removing fewer than $5 \%$ of the substations can leave half the remaining grid without service.

\subsection{Topological Influence on Serviceability}

Removing one client is always at least a damaging to serviceability as removing one link, so it makes sense that client removal is more damaging than link removal in both the Wireless Mesh and Power Grid networks. But the Wireless Mesh Network exhibits profound robustness even after a relatively large quantity of links have been removed while the Power Grid does not. Upon examination we find that clients in the former have a much more rich degree distribution than clients in the later. Even though the Wireless Mesh Network has just

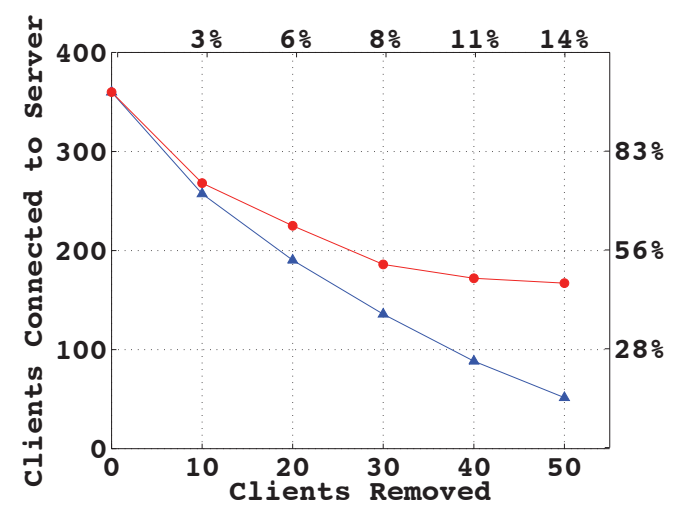

Figure 5: Power Grid connectivity (substation removal)

$20 \%$ of the clients as the Power grid, more than half the clients in the Wireless Mesh network share a link with at least eight other nodes. In contrast, more than half the nodes in the Power Grid share four or fewer links with adjacent nodes. We believe that this dramatic difference in degree distribution between the two networks is what develops the significant difference in robustness.

\section{AN ALGORITHM FOR LARGE GRAPHS}

For large graphs, even the branch-and-cut procedure MinConnBC will fail because the main component MinConnSDP itself either fails to converge or returns an extremely weak bound. In this section, we provide an additional solution to bound $\operatorname{Min} \operatorname{Conn}(G, n, 0)$ that instead uses MinConnSDP as a subroutine in a divide-andconquer algorithm. Our approach is the first capable of providing both upper and lower bounds on connectivity after vertex removal in graphs with thousands of vertices. The limitation of this extension is that our analysis does not apply to edge cuts and it will not work well for every graph. Success is very topologically dependent.

\subsection{A Divide-and-Conquer Solution for Client Removal}

Let $G$ be a client-server graph. Our strategy is to solve $\operatorname{Min} \operatorname{Conn}(G, n, 0)$ by breaking $G$ into subgraphs $G_{1}, \ldots$ by groups of clients that act as gateways for other clients. The connectivity properties of subgraph $G_{i}$ can be found by solving a series of subproblems of the form $\operatorname{Min} \operatorname{ConnBC}\left(G_{i}, n_{i}, 0\right)$. Once the connectivity properties of each subgraph $G_{i}$ have been quantified, the solution to $\operatorname{Min} \operatorname{Conn}(G, n, 0)$ is formed by judiciously choosing those subproblems that render the lowest clientserver connectivity.

Our objective is to count the minimum number of clients that remain connected to at least one server after the removal of some fixed number of clients. Therefore, we seek to count the minimum number of clients that retain a service path after the removal of some fixed 
number of other clients. Generally, the serviceability of a client can depend on the serviceability of every other client; but in real world networks, this is rarely the case. We begin by describing how to isolate the serviceability of a subgraph in an arbitrary client-server graph.

For arbitrary $G=(S \cup C, E)$ define the set of gateways, $Y \subseteq C$, as those clients that are adjacent to at least one server in $S$. The remaining clients are labeled children are placed in the child set $L$. A subset of children, $L^{\prime}$, are said to be siblings when they form a single connected component after all gateways are removed. For each set of siblings $L^{\prime}$, there is a corresponding subset of gateways, $Y^{\prime}$ that are adjacent to the children in that set. We call such a subset $Y^{\prime}$ the gateways of siblings $L^{\prime}$ and conversely, we call siblings $L^{\prime}$ the children of gateways $Y^{\prime}$. Together, the set $Y^{\prime} \cup L^{\prime}$ is called a family. The serviceability of the children in a family depends entirely on their connectivity to the gateways of that family.

OBSERVATION: If $F$ is a family in $G$ and $c$ is a child in $F$, then $c$ is serviceable iff there is a path from $c$ to some gateway in $F$.

According to the above observation, we can decouple the serviceability of clients in different families. This leaves us with a divide-and-conquer strategy for solving $\operatorname{Min} \operatorname{Conn}(G, n, 0)$ as Knapsack Problem.

\section{PROCEDURE: $\operatorname{Min} \operatorname{ConnDC}(G, n, 0)$}

Let $G$ be a client-server graph. Bound the solution to Problem $\operatorname{Min} \operatorname{Conn}(G, n, 0)$ as follows.

- Form an exhaustive and disjoint decomposition of $G$ into families $\mathcal{F}=F_{1}, F_{2}, \ldots$

- Form the following knapsack-like subproblem. To each family $F_{i}=\left(Y_{i}, L_{i}\right)$, assign a maximum weight $W_{i}=\left|Y_{i}\right|$ and unit value multiplier $U_{i}$ so that for any quantity, $x$, of removed clients in $F_{i}$, the value $x U_{i}$ bounds from below the number of clients that are not serviceable.

- Solve the subproblem for maximum aggregate weight $n$ where item $i$ corresponds to family $F_{i}$, with weight $x \in\left[0, W_{i}\right]$ and value $x U_{i}$. An upper bound is readily available by means of greedy choice according to highest unit value. The lower bound is furnished by Linear Program Select $L P(W, U, n)$.

PROGRAM: Select $L P(\mathrm{~W}, \mathrm{U}, \mathrm{w})$

- Given: weight and value multiplier vectors $W$ and $V$, and maximum aggregate weight $w$.

- Find: maximum $\sum_{i} X(i) U(i)$ such that

(1) $\sum_{i} X(i) \leq w$.

(2) $X(i) U(i)-W(i) \leq 0, \forall i$.

(3) $X(i) \in\{0, W(i)\}, \forall i$.
Next, we derive the maximum weight and unit value multiplier functions for an arbitrary client-server graph $G$. The first step is to decompose $G$ into exhaustive and disjoint families. We begin by identifying all families in $G$, then independently measure how their serviceability is affected by client removal, and finally combine the results for each family. Algorithm 1 addresses the first task for client-server graph $G=(S \cup C, E)$. In this algorithm, subroutine NEIGHBORS $(G, c)$ returns the set of vertices in $G$ that share an edge in $E$ with vertex c. Subroutine COMPONENTS $(G)$ returns a set of sets of vertices, with each set of vertices corresponding to a connected component of $G$. Subroutine CLOSURE $(\mathcal{F})$ returns the transitive closure of the union operation on each set $F \in \mathcal{F}$ - i.e., subroutine $\operatorname{CLOSURE}(\mathcal{F})$ combines overlapping sets in $\mathcal{F}$ until all families in $\mathcal{F}$ are pair-wise disjoint.

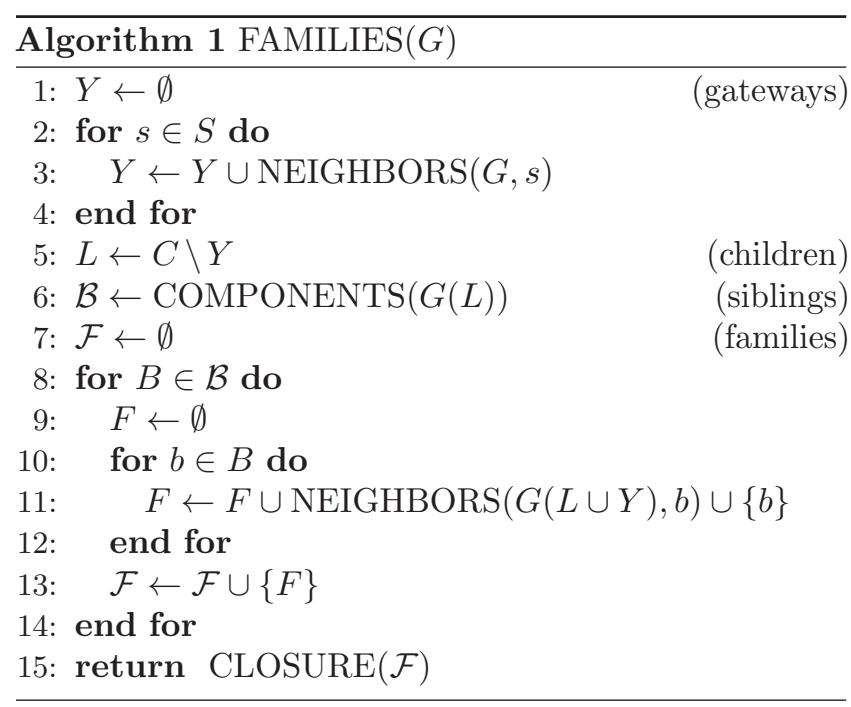

Lines 1-4 are devoted to delineating two sets $Y$ and $L$, which contain all gateways and children respectively. In line 6, families of $G$ are initially formed as the connected components of the subgraph of $G$ induced by the set of all children $L$. In lines 8-14, each family is enlarged to include the gateways for each set of siblings. Finally, in line 15 , we combine families that share common gateways so that each family is disjoint from every other family.

Let $\mathcal{F}$ be the set of all families in $G$ as constructed by Algorithm 1. We next seek to characterize the serviceability of each family $F \in \mathcal{F}$ by a pair of numbers: the maximum weight and the unit value multiplier, which we label $W(F)$ and $U(F)$ respectively. The maximum weight will be equal to the number of clients that must be removed in order to completely disconnect $F$ from every server, and the unit value multiplier will be formulated so that $x U(F)$ bounds from above the number of non-serviceable clients in $F$ after the removal of any $x$ clients. The maximum weight of family $F$ is always equal to the number of gateways in $F$ because on the one 


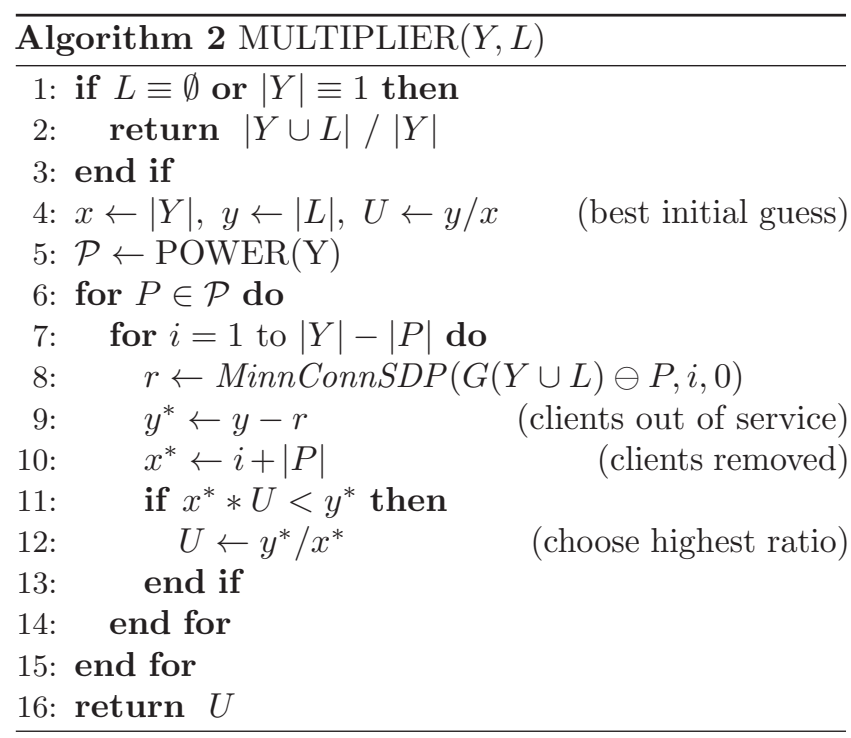

hand, there is no way to indirectly disconnect a gateway from all servers (which bounds $W(F)$ from below), and on the other hand, every child in $F$ must ultimately contain at least one gateway on every service path (which bounds $W(F)$ from above). Constructing the unit value multiplier is more difficult because there are many ways to remove clients in $F$ and we must take care to find the most damaging combination for each quantity on the interval $[1, \ldots, W(F)]$. Algorithm 2 shows how to construct $U(F)$ for an arbitrary $F$ and according to our requirement that $U(F)$ yield an upper bound on non-serviceability. The subroutine POWER $(Y)$ returns the power set of set $Y$.

Algorithm 2 begins by testing for trivial cases where we can immediately identify the unit value multiplier. For all other cases (line 4), we begin by setting the multiplier to the most obvious ratio where all gateways are removed. It is possible that a better solution exists, and by iterating on all possible subproblems of this family, we progressively refine this choice of unit value multiplier. Line 8 finds the solution to each subproblem by running it through $\operatorname{Min} \operatorname{Conn} \operatorname{SDP}(G, n, 0)$. Note that in Section 2, we worked with relatively large graphs that did not immediately yield a strong solution to the semidefinite program, and therefore required Procedure MinConnBC for strengthening the result. For the subproblems in Algorithm 2, however, we find that the problems are small enough that further analysis is not necessary. The solution to $\operatorname{Min} \operatorname{ConnSDP}(G, n, 0)$ gives a lower bound on the number of clients that remain serviceable after removing some $n$ clients. For $y^{*}$, however, we require an upper bound on the total number of clients that are removed from service after removing $n$ clients. Line 9 reverses the output from MinConnSDP from number of clients connected to number disconnected. The remaining lines replace the old multiplier value only if the new value is greater.

\subsubsection{Managing Subproblem Size}

Algorithm 2 iterates over the power set of $Y$. This set becomes quite large for even small sets, so we aim to keep the number of gateways at 5 or fewer. However, we continue to require that families remain pair-wise disjoint, so we must find a way to split families with large gateway sets. For the purpose of creating a lower bound on $\operatorname{Min} \operatorname{Conn}(G, n, 0)$, it's clear that removing edges will only lower the value of the solution and will not jeopardize the bound. So, we judiciously choose edges to remove so that there are 5 or fewer gateways corresponding to each family in the new graph. Since we are dealing with relatively small subgraphs, a simple spectral approach will suffice.

Ding et. al [14] showed how to use the eigenvector corresponding to the second smallest eigenvalue of the Laplacian Matrix of a graph to find nearly-disconnected components of that graph. Consider the graph $H=$ $G(F)$ for any family $F$. The spectrum of the Laplacian of $H$ has one eigenvalue equal to 0 for each connected component in $H$. In this case, zero-valued eigenvalues are the smallest because the Laplacian is always real and symmetric so its eigenvalues are always non-negative. Any eigenvector belonging to a 0 eigenvalue will have the same value for all indices that correspond to vertices in the same connected component. Matrix perturbation theory predicts that graphs that have nearly disconnected components will show similar eigenvector values between indices corresponding to vertices in each component when considering the eigenvector corresponding to the second smallest eigenvalue. The following procedure outlines the steps that we took to to break families with large gateway sets into groups of families with no more than 5 gateways.

Let $F=(Y, L)$ be a family in client-server graph $G$ and define subroutine $\operatorname{EIGENVECTOR}(H)$ to be a function that returns the eigenvector corresponding to the second smallest eigenvalue of the Laplacian of $H$. If $|Y|>5$, it can be disassembled with the following steps.

- Compute $x \leftarrow \operatorname{EIGENVECTOR}(G(F))$

- Let $k=\lceil|Y| / 5\rceil$, and break $Y$ into $k$ subsets labeled $Y_{1}, \ldots, Y_{k}$ where $\left|Y_{i}\right|=5$ for $i<k$ and $\left|Y_{k}\right|=$ $|Y|-5(k-1)$.

- Assign clients of $Y$ to one of the subsets so that clients in the same subset have similar entries in eigenvector $x$.

- Create $k$ new families $F_{1}, \ldots, F_{k}$ so that family $F_{i}$ has set $Y_{i}$ as its gateways, and create child sets $L_{i}$ for each family $F_{i}$ with $L_{i}$ initially empty.

- Assign each child in $L$ to the family $F_{i}$ whose average eigenvector entry matches closest to its own eigenvector entry. 


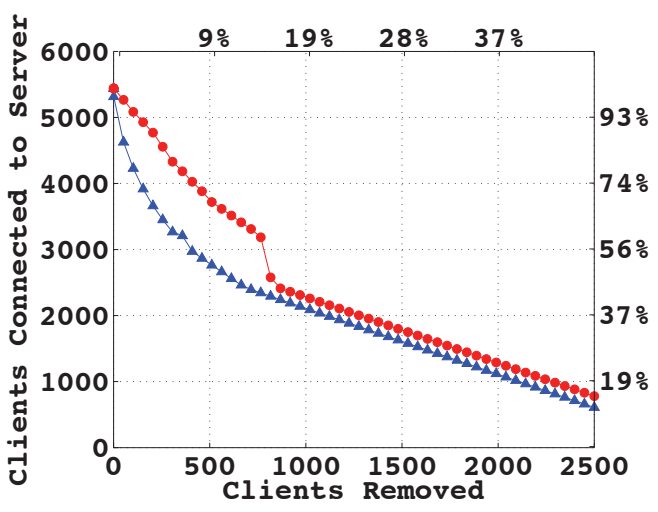

Figure 6: AS graph connectivity

\subsection{Evaluation on the Internet AS Graph}

Our first evaluation is on a snapshot of the Internet at the Autonomous Systems (AS) level taken by the CAIDA project in April 2005 [13]. Their taxonomy identifies inferred Tier-1 and Tier-2 ASes. We create a client-server graph with Tier-1 and Tier-2 ASes labeled as servers and clients, respectively, and with an edge set derived from the corresponding list of peering relationships. We assume that the Tier-1 AS Internet backbone remains fully intact, but that Tier-2 ASes are vulnerable to removal due to attack or administrativelevel disenfranchisement. We don't claim that this is the most likely attack on the Internet; we present it as a means of understanding how node removal at the edge of the Internet influences overall connectivity.

Let $G$ denote the client-server graph that models the AS graph. We use MinConnDC to compute upper and lower bounds on $\operatorname{Min} \operatorname{Conn}(G, n, 0)$ for various values of $n$. Figure 6 shows the results where the number of clients removed $(n)$ varies from 0 to 2,500 in increments of 50. The blue curve in triangles is the lower bound delivered as the solution to our knapsack problem. The red curve in circles is an upper bound developed by actually removing from $G$ those client vertices identified by the knapsack solution.

In contrast to the performance of $\operatorname{Min} \operatorname{Conn} B C(G, n, 0)$ from Section 2, the Knapsack solution has somewhat weak performance for small values of $n$, but tightens considerably for larger values. The initial weaker performance can be attributed to the error introduced by splitting families with large gateway sets. Algorithm 1 returned 2,759 families total, and among those there were 9 that had more than 5 gateways vertices. The largest set was found in one family that had 292 gateways. The rest had fewer than 30 gateways. By splitting the largest gateway set almost 60 times we introduced error. Unfortunately, it's difficult to predict a priori how much error will be introduced by splitting families with large gateway sets.
The lower bound makes it seem easier to disconnect service paths to a larger number of clients than is actually possible. However, once $n$ is large enough that this large gateway set can actually be removed, the upper bound settles close to the lower. We believe that this type of tradeoff will be typical when applying $\operatorname{Min} C o n n D C$ to any client-server graph with such a hierarchical structure. Accuracy will largely depend on the extent to which the graph can be decomposed into families with the small gateway sets.

In our analysis we identified a small handful of families responsible for almost all of the complexity in the graph. They had large gateways sets and large sets of children. The remaining families had small gateway sets and small sets of children. They appear as commodities to the knapsack algorithm. Overall, the AS graph exhibits strong serviceability in the face of significant deterioration of Tier-2 clients. Even when 900 (>16\%) Tier-2 clients are removed, better than half the Internet remains in service. This result appears in sharp contrast to research outlined in Section 1.3 where both Tier 1 and Tier 2 nodes can be removed. There, far fewer than $50 \%$ of all nodes remain in the connected component even when only $3 \%$ of the nodes have been removed $[22,34]$.

\subsection{Evaluation on Airport Connectivity}

We next consider airport connectivity in the states of Iowa and Michigan. To do so, we began by obtaining U.S. highway information from the Oak Ridge National Laboratory [2]. These were found in a single large file composed of more than $200 \mathrm{~K}$ different routes in North America. Each route was delineated by a sequence of coordinates. We call these coordinates waypoints. From the Socioeconomic Data and Applications Center (SEDAC) [1], we obtained population density estimates from the 2000 Census that are accurate to within several hundred meters. We chose airports as only an example of critical infrastructure, and as in our above cases, there are limitations to our analysis. We make the simplifying assumption that each airport is equally resourceful. We also do not claim this is the most effective attack on infrastructure, but it does server to bound the severity of a particular kind of coordinated attack on a very import piece of national infrastructure.

For each state, we extracted the routes and corresponding waypoints that fall within the bounding box containing that state. A graph representing the highway connectivity of each state was then generated by creating a client vertex for each waypoint and an edge for any two adjacent waypoints in a given route. Because one route will often end at the beginning of another, we consolidated waypoints that were within 100 meters of each other into a single vertex. Our next step was to create a server vertex for each airport, which we linked to all waypoints within a (roughly) six-mile radius. The 


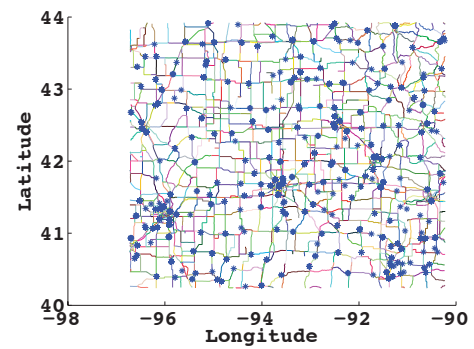

(a) Iowa

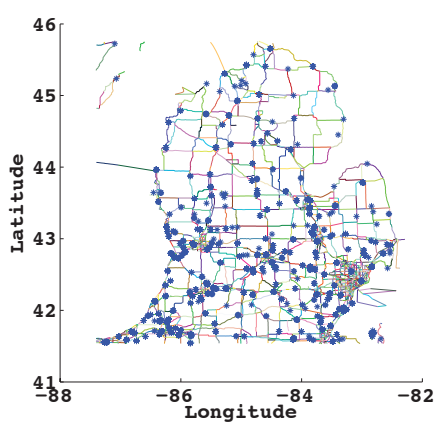

(b) Michigan
Figure 7: Airports are shown as blue dots over highways in (a) Iowa and (b) Michigan. Colors vary between different routes. Waypoints are not shown.

coordinates for airports in these states were found at the data warehouse site socrata.com [5]. The final step in graph construction was to assign a value to each waypoint corresponding to the estimated population density near that point using the SEDAC data. Since people might arrive at an initial waypoint near their place of residence by means other than automobile, we averaged the measured population density between all waypoints within approximately 15 miles of each other and assigned each waypoint a value corresponding to this population density. This means that the sum of all waypoint values in a given state is approximately equal to the population of that state. Figure 7 shows all the routes used for each state as well as the available airports.

Our construction yielded a client-server graph with clients corresponding to waypoints in the given state and whose value was approximately equal to the portion of the state's population residing near the waypoint. Our objective was to bound the quantity of people who maintain highway access to any airport after the removal of some fixed number of waypoints. We imagine that the waypoints are removed by either natural disaster of coordinated attack. We constructed a solution to the WeightedMinConn Problem for this scenario by modifying MinConnDC as follows: i) client values were incorporated by first modifying Algorithm MULTIPLIER to return the highest ratio of client values to gateways removed, ii) the objective function of Program Min$C o n n S D P$ was altered to minimize the aggregate value of connected clients.

\subsubsection{Iowa}

To apply our WeightedMinConn solution, we constructed a client-server graph corresponding to the state of Iowa. The boundary of the state was delineated by the coordinates $(-104.3701,42.6738)$ and $(-96.2842,46.1566)$. In total there were 1947 waypoints and 272 airports.

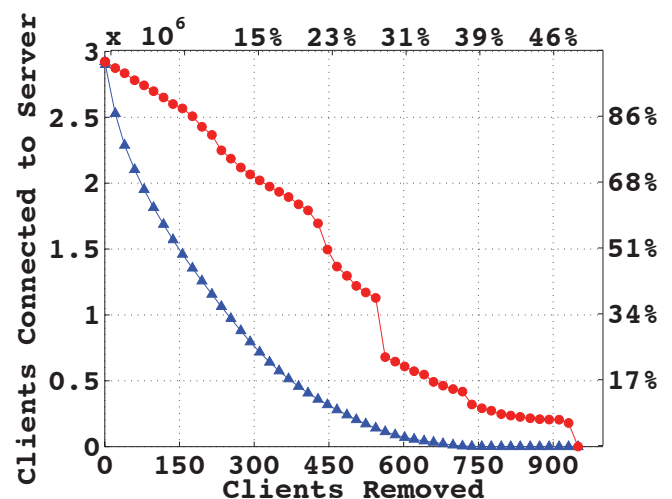

Figure 8: Airport connectivity in Iowa

Algorithm FAMILIES returned 493 families total with just three families having more than 5 gateways. They had gateway sizes 8,19 , and 501. Breaking the family with 501 gateways into 101 subfamilies was the main source of error. It causes the lower bound to retreat substantially from the upper for small numbers of removed waypoints.

Figure 8 shows that despite the somewhat loose agreement between upper and lower bounds, we can still draw interesting conclusions about how airport access degrades with waypoint removal. For example, when just 200 waypoints (approximately 10\%) are blocked, it is possible to disconnect at least $500 \mathrm{~K}(15 \%)$ people from every airport, but no more than $1.7 \mathrm{M}(50 \%)$ can possibly be disconnected. There is also a sudden drop in the upper bound after approximately 575 waypoints are removed. This is the point when the largest family (the one that began with 501 gateways) is finally completely disconnected. It's a critical point where the number of people connected drops by about $500 \mathrm{~K}$.

\subsubsection{Michigan}

We performed similar analysis for the state of Michigan where the corresponding client-server graph contained 2207 waypoints and 285 airports. We chose to focus on the lower peninsula of Michigan (the mitten) bounded by the coordinates $(-87.4072,41.5327)$ and (-82.2656,45.7593). After applying Algorithm FAMILIES, there were 806 families total with 15 having more than 5 gateway vertices. The three largest gateway sets were 129, 112, and 53. All other families had fewer than 25 gateways. Figure 9 shows how airport connectivity degraded with waypoint removal. Again, the families with large gateway sets were the major source of error in the lower bound. They forced a large gap between upper and lower bounds for up to 300 removed waypoints. Subsequently, the bounds tightened significantly.

From the plot we can see that no more than 4.3 million people (43\%) will maintain airport connectivity after 300 waypoints have been removed, and it's possible that as 


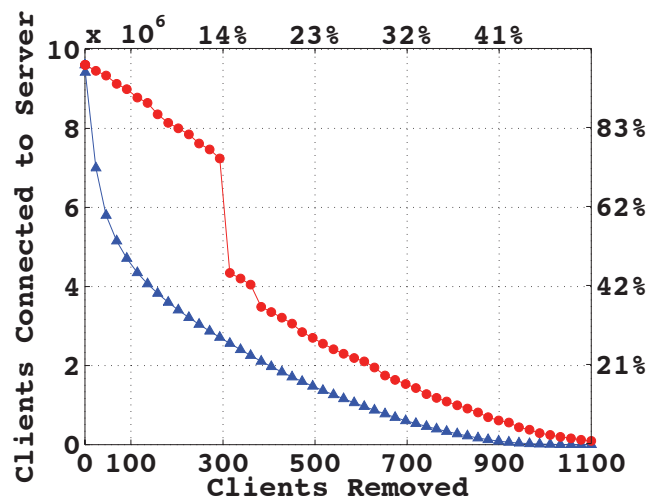

Figure 9: Airport connectivity in Michigan

few as 2.8 million (28\%) will maintain connectivity. In other words, at least half the residents of Michigan will lose airport access if fewer than $14 \%$ of the waypoints are removed.

\section{CONCLUSION}

We have introduced an intuitive model, the clientserver graph, that is capable of describing many realworld networks more richly than its predecessors. We have also introduced the Problem MinConn, which attempts to quantify the minimum number of client vertices connected to a server after either edge or client removal. Our framing of, and solution to, this problem is preferable to existing techniques, such as localized heuristics and graph separators, because it offers concrete bounds for edge, vertex and mixed edge-vertex removal in any graph, and it also differentiates between resource providers (servers) and consumers (clients). Our methods are also easily extended to find the minimum aggregate weight of serviceable clients after weighted client removal. Finally, our solution leverages a graph's topology to provide bounds for large graphs, a domain where other techniques often fall short.

We used our solution to MinConn to demonstrate how client serviceability degrades in various real-world networks. This analysis enabled us to see that the sparse connectivity of the Philippine Power Grid makes it much more susceptible to link attack than the relatively dense Net Equality Wireless Mesh Network. We also saw that the Internet AS level graph is reasonably robust to attacks on the edge of the network. Lastly, we showed that coordinated attacks on the highway systems in Iowa and Michigan could impose large-scale limitations on personal mobility.

\section{REFERENCES}

[1] http://sedac.ciesin.columbia.edu.

[2] http://www-cta.ornl.gov/transnet/Highways.html.

[3] http://www.haciendacdc.org.

[4] http://www.ngcp.ph/gridmap/2k6gridmap.pdf.

[5] http://www.socrata.com.
[6] M. Amin. Security challenges for the electricity infrastructure. IEEE Computer, 35(4):8-10, Aug 2002.

[7] M. Amin. North America's electricity infrastructure: are we ready for more perfect storms? IEEE Security 83 Privacy, $1(5): 19-25,2003$.

[8] S. Aref and B. Miller. Damage Information and Reporting Tool. Technical report, Common Ground Alliance, 2005.

[9] C. Berrick, S. Morris, and G. Malavenda. Federal highway report. Technical report, USGAO, 2009.

[10] G. D. Bissias, B. N. Levine, and R. K. Sitaraman. Finding Weak Points in Networks. Technical Report UM-CS-2010-037, UMass Amherst, 2010.

[11] T. Bui and C. Jones. Finding good approximate vertex and edge partitions is NP-hard. Info. Proc. Lett., 42(3), 1992.

[12] R. Cohen, K. Erez, D. ben Avraham, and S. Havlin. Breakdown of the Internet under Intentional Attack. Phys. Rev. Lett., 86(16):3682-3685, Apr 2001.

[13] X. Dimitropoulos, D. Krioukov, G. Riley, and K. Claffy. Revealing the Autonomous System Taxonomy. In Proc. PAM Conference, March 2006.

[14] C. Ding, X. He, and H. Zha. A spectral method to separate disconnected and nearly-disconnected web graph components. In Proc. ACM SIGKDD, pages 275-280, 2001.

[15] A. Flaxman, A. Frieze, and J. Vera. Adversarial deletion in a scale free random graph process. In Proc. ACM-SIAM SODA, pages 287-292, 2005.

[16] S. Gorman, L. Schintler, R. Kulkarni, and R. Stough. The revenge of distance. Jrnl Cont. and Crisis Mgmt. 12(2):48-63, 2004.

[17] J.-L. Guillaume, M. Latapy, and C. Magnien. Comparison of Failures and Attacks on Random and Scale-Free Networks. In Proc. OPODIS, pages 186-196, Dec 2004.

[18] C. Helmberg. Semidefinite programming. European Journal of Operational Research, 3(137):461-482, 2002.

[19] A. Hickson. Terrorist Threat to U.S. Highway System. Technical report, U.S. DHS, June 2006.

[20] S. Kent, C. Lynn, J. Mikkelson, and K. Seo. Secure Border Gateway Protocol (S-BGP). In Proc. ISOC NDSS, Feb 2000.

[21] Y. Law et al. Energy-efficient link-layer jamming attacks against wireless sensor network MAC protocols. In Proc. $A C M S A S N$, pages 76-88, 2005.

[22] D. Magoni. Tearing down the Internet. IEEE JSAC, 21(6):949-960, 2003.

[23] S. Murphy. BGP Security Vulnerabilities Analysis. Technical report, IETF draft, February 2002.

[24] A. Perrig, J. Stankovic, and D. Wagner. Security in wireless sensor networks. Commun. ACM, 47(6):53-57, 2004.

[25] K. Poulsen. The Backhoe: A Real Cyberthreat. Wired magazine, Jan 2006.

[26] P. Pourbeik, P. Kundur, and C. Taylor. The anatomy of a power grid blockout. IEEE Power \& Energy Magazine, 4(5):22-29, 2006.

[27] A. Rosenberg and L. Heath. Graph Separators with Applications. Springer, 2001.

[28] B. Smith and J. J. Garcia. Securing the Border Gateway Routing Protocol. In Proc. Global Internet, Nov. 1996.

[29] D. Watts. Security and vulnerability in electric power systems. In Proc. North American Power Symp, pages 559-566, Oct 2003.

[30] H. Wolkowicz and Q. Zhao. Semidefinite Programming Relaxations for the Graph Partitioning Problem. Discrete Applied Mathematics, 96-97:461-479, 1999.

[31] W. Xu, W. Trappe, Y. Zhang, and T. Wood. The Feasibility of Launching and Detecting Jamming Attacks in Wireless Networks. In Proc. ACM Mobihoc, pages 46-57, May 2005.

[32] H. Yang, H. Luo, F. Ye, S. Lu, and L. Zhang. Security in mobile ad hoc networks: challenges and solutions. Wireless Communications, IEEE, 11(1):38 - 47, Feb. 2004.

[33] S. Zhou and R. Mondragon. Redundancy and robustness of AS-level Internet topology and its models. Electronics Letters, 40(2):151-152, 2004. 